\title{
Pyoderma gangrenosum in a patient with common variable primary immunodeficiency
}

\author{
Beata Bergler-Czop, Ligia Brzezińska-Wcisło \\ Department of Dermatology, Silesian Medical University, Katowice, Poland \\ Head: Prof. Ligia Brzezińska-Wcisło MD, PhD
}

Postep Derm Alergol 2013; XXX, 3: 188-191

DOI: $10.5114 /$ pdia.2013.35622

\begin{abstract}
Pyoderma gangrenosum is a rare inflammatory skin condition that is associated with systemic inflammatory diseases. It is characterised by the presence of well-secluded, painful ulcerations, often located on the lower limbs. Similarly as in the case of acne inversa, patients' markers of the inflammatory process are elevated; including OB, $\mathrm{C}$-reactive protein and leukocytosis. The aetiology takes into account an over-reactive inflammatory response to various factors (presence of the so-called pathergy symptom). Common variable primary immunodeficiency (CVID) is a disease that is rather often recognized and affects about 1/10,000-100,000 individuals. It is a heterogeneous group of disorders of combined B-and T-cell dysfunction. The case is described of a 22 -year-old man with pyoderma gangrenosum that coexisted with CVID.
\end{abstract}

Key words: pyoderma gangrenosum, immunodeficiency, inflammation.

\section{Introduction}

Pyoderma gangrenosum is a chronic disease with inflammatory aetiology. It is believed that the role here is played by the dysregulation of the patient's immune system and the production of pro-inflammatory cytokines, mainly tumor necrosis factor- $\alpha$ (TNF- $\alpha$ ) and interleukin 8 , 16 , and $1 \beta$ (IL-8, IL-16 and IL-1 $\beta$ ) $[1,2]$.

Pyoderma gangrenosum is characterised by the presence of well-secluded, painful ulcerations, often located on the lower limbs. Similarly as in the case of acne inversa, patients' markers of the inflammatory process are elevated; including $\mathrm{OB}, \mathrm{C}$-reactive protein and leukocytosis. The aetiology takes into account an over-reactive inflammatory response to various factors (presence of the socalled pathergy symptom). However, the relationship with an underlying disease is most often described: inflammatory bowel diseases (mainly Crohn's disease), liver diseases (HCV, immune hepatitis), rheumatic and hematologic diseases (monoclonal gammopathies, leukaemias). There are also reports of cases of post-traumatic pyoderma gangrenosum (including those in surgical scars), and pyoderma gangrenosum associated with sarcoidosis, solid tumours, HIV/AIDS, conglobated acne and inverted acne [3].
Common variable primary immunodeficiency (CVID) is a disease that is rather often recognized and affects about $1 / 10,000-100,000$ individuals. It is a heterogeneous group of disorders of combined B-and T-cell dysfunction. Some cases could be asymptomatic, but in most patients severe clinical manifestations occur, such as recurrent sinusitis, recurrent pneumonia, bronchiectasis, lymphadenopathy and an increased lifetime risk of lymphoma and autoimmune disease. For diagnosis, marked reduction in serum levels of IgG (or IgG subclasses) +/-IgA deficiency is typical. Approximately $50 \%$ of patients have also associated IgM deficiency. In treatment, the replacement therapy with intravenous immunoglobulin every 3-4 weeks is used [4].

The cases of pyoderma gangrenosum that coexist with immunodeficiency are relatively seldom presented.

\section{Case report}

The patient is 22 years old. The first skin lesions in the form of a quickly spreading peripherally ulcer on the left lower leg occurred a year ago. Since he was one, the patient has been under control of the Department of Allergology and Immunology due to recognised common primary variable immunodeficiency. He has also suffered from recurrent infection of the upper and lower respiratory tract. Recur-

Address for correspondence: Beata Bergler-Czop MD, PhD, 2a Lesna St, 42-624 Ossy, Poland, phone/fax: +48 322840877 , e-mail: bettina2@tlen.pl

Received: 16.09.2012, accepted: 10.02.2013. 
rent sinusitis and bronchiectasis were also diagnosed. In treatment, replacement therapy with intravenous immunoglobulin every 3-4 weeks has been used. The patient also took antibiotics several times. The patient did not take any medication permanently for any other reasons, and did not suffer from other chronic diseases. In the family history, no immunodeficiency was diagnosed.

In 2011, in the histopathological examination of the edge of the lesion on the left lower leg, features of vasculitis with massive leukocyte infiltrates, presence of plasma cells, histiocytes and giant cells were found. The whole picture was not diagnostic.

At that time the patient was treated in the outpatient clinic. It was only local treatment. Because of no improvement and massive overgrowth of the granulatio that could suggest neoplastic transformation, the man was referred to the Department of Dermatology, Silesian Medical University.

On admission there was an ulcer on the left lower leg. On the medial posterior surface of the left lower leg there was an oval ulcer of $10 \mathrm{~cm} \times 15 \mathrm{~cm}$, with undermined rolled edges and the bottom covered with large amounts of purulent secretions, necrotic tissues and granulatio. The skin surrounding the whole ulcer was red, very warm, with features of inflammation. Peripheral lymph nodes were not enlarged. Mucous membranes were free from lesions (Figure 1).

Laboratory tests: ESR 35, CRP 11.3, WBC 10.9, glucose, full blood count, electrolytes, AST, ALT, GGTP, bilirubin, creatinine, urea, general urine test, electrophoresis, CPK, aldolase, Latex - R, Waaler-Rose's reaction were normal. Protein of the complement: C3 $172 \mathrm{mg} / \mathrm{dl}(\mathrm{N}), \mathrm{C} 4-61 \mathrm{mg} / \mathrm{dl}$ (10-40), lgA - 6 mg/dl (70-400), lgG - 655 mg/dl (700-1600), lgM-13 mg/dl (40-230). Tumour markers: CEA, CA 125, total PSA were within the norm. Imaging: $X$-ray of the chest, left lower leg, ultrasonography of the abdomen were normal. Mycology - negative. Histology - an edge of the ulcer - pyoderma gangrenosum. Proliferatio reactiva epitheli plani; the bottom of the lesion - necrosis et granulatio. Vasculitis. Immunological consultation - without contraindication to cyclosporine, but this kind of therapy needs higher doses of intravenous immunoglobulin (Kriovig) and regular control of the levels of immunoglobulin and proteins of the complement.

During his stay at the hospital, methylprednisolone was applied at a dose of $24 \mathrm{mg} /$ day, cefuroxime at a dose of 2.0 and the local treatment. Because of the result of immunological consultation and fast improvement of the local stage, we stopped using cyclosporine. After his local and general symptoms improved, the patient was transferred to the Department of Allergology and Immunology in order to continue treatment with immunoglobulin. Dermatological treatment is continued at the Outpatient Clinic of Dermatology, where healing of the ulcer is observed and the dose of methylprednisolone is slowly tapered.

\section{Discussion}

The aetiology of pyoderma gangrenosum is unclear. It is believed that dysregulation of the patient's immune system plays a role here and that is why it could coexist with the immunology diseases. However, the cases associated with immunodeficiency (especially congenital) are relatively rarely described in the literature [5]. The relationship with an underlying disease is most often described: inflammatory bowel diseases (mainly Crohn's disease), liver diseases (HCV, immune hepatitis), rheumatic and hematologic diseases (monoclonal gammopathies, leukaemias) [1, 6].

Therapy of pyoderma gangrenosum, regardless of its aetiology, is difficult. In treatment we can use general glucocorticosteroids, dapsone, clofazimine, cyclosporine A, tacrolimus, mycophenolate mofetil, intravenous immunoglobulin, anti-TNF inhibitors and monoclonal antibodies [1]. The therapy of our patient, because of his basic disease, had some limitations.

Neiderer et al. [7] presented a 76-year-old male patient with a history of rheumatoid arthritis and recalcitrant pyoderma gangrenosum. After 9 months of treatment with local wound care, steroids, and topical tacrolimus, the wound had increased in size. At that time, he was on a regimen of five applications of a bioengineered cell-based product with twice-weekly mechanically powered negative pressure device changes and $40 \mathrm{mg}$ of prednisone. The wound was completely healed after 16 weeks. Also Fraccalvieri et al. [8] described a good medical result of treatment of pyoderma gangrenosum with a negative pressure device.

Andrisani et al. [10] suggest the efficacy of infliximab (inhibitor TNF- $\alpha$ ) in the treatment of pyoderma gangrenosum in the case of a patient with ulcerative colitis and

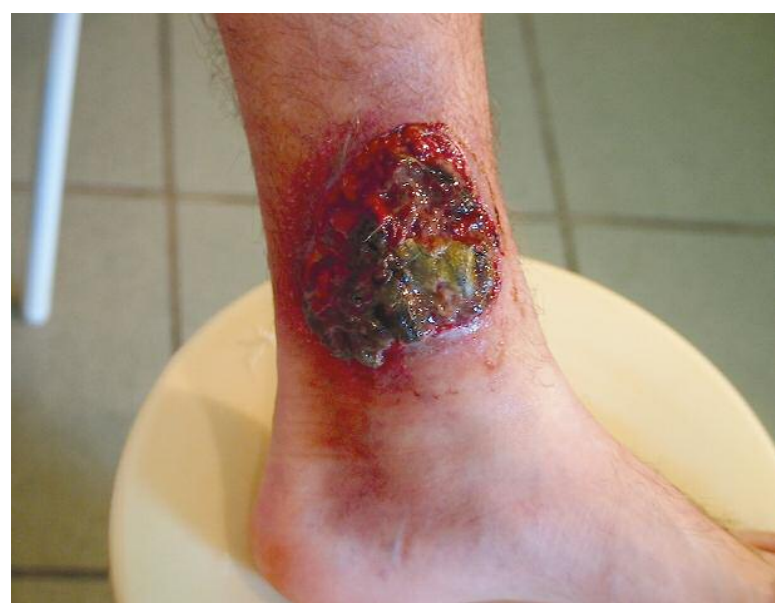

Fig. 1. 22-year-old patient: on the medial posterior surface of the left lower leg there is an oval ulcer of $10 \mathrm{~mm} \times 15 \mathrm{~cm}$, with undermined rolled edges and the bottom covered with large amounts of purulent secretions, necrotic tissues and granulatio 
an ulcer localized on the left breast. Also in a patient of Mooji et al. [11] pyoderma gangrenosum was treated with infliximab with good results. Biological treatment (etanercept) was also used by Kim et al. [12].

Pyoderma gangrenosum could often be difficult to diagnose and could be suspected of neoplastic changes. Spinocellular carcinoma was also suggested in the case described by us. However, histopathological examination confirmed the diagnosis of pyoderma gangrenosum.

Wolfe et al. [13] described a case of atypical pyoderma gangrenosum of the dorsal hand in a patient who presented with the histopathologic and clinical diagnosis of squamous cell carcinoma (SCC). Atypical pyoderma gangrenosum is a rare variant of pyoderma gangrenosum that occurs on the upper extremities, presenting as a cutaneous ulcer. The correct treatment is nonsurgical, and surgical intervention often results in trauma-induced expansion of lesions.

As mentioned, the cases of pyoderma gangrenosum that coexist with immunodeficiency are relatively seldom presented. More often some patients with HIV are presented. Maksimovic et al. [14] presented a 53-year-old male treated since 1989 for HIV infection who had been presenting two neutrophilic diseases, that could be connected with immunodeficiency: erythema elevatum diutinum and pyoderma gangrenosum. Kreuter et al. [15] described a patient with pyoderma gangrenosum and psoriasis also in the course of HIV infection.

Since he was one, our patient has been under control of the Department of Allergology and Immunology because of recognised primary variable immunodeficiency. However, in treatment, replacement therapy with intravenous immunoglobulin every 3-4 weeks is used in our patient, and we observed progression of skin changes. Differently from the patient of Nord et al. [16] - a 31-year-old Caucasian male with leukocyte adhesion deficiency I and a 20-year history of pyoderma gangrenosum, where upon completion of six courses of intravenous immunoglobulin, ulcerations had nearly healed for the first time in a decade.

Hinze et al. [17] reported an 11-year-old boy with a leukocyte adhesion deficiency type 1 and longstanding history of recurrent pyoderma gangrenosum. This article emphasizes the importance of considering immunodeficiencies in the differential diagnosis of patients with recurrent skin ulcers. Elenberg et al. [18] presented a patient with pyoderma gangrenosum after bone marrow transplantation for leukocyte adhesion deficiency type 1. Bedlow et al. [19] described a 5-year-old girl with a history of recurrent bacterial infections since early childhood who developed necrotic skin ulcers - pyoderma gangrenosum and a persistent circulating neutrophilia. Histologically, the lesions showed deep ulceration with a diffuse lymphohistiocytic infiltrate, but with a relative scarcity of neutrophils. Subsequent investigation revealed a complete absence of CD11a/CD18 beta 2 integrins on the surface of the patient's neutrophils, confirming the diagnosis of LAD type 1 .
Paller et al. [20] presented two children with congenital immunodeficiency in whom pyoderma gangrensum developed. In a 60-year-old patient of Carsuzaa et al. [21] with a history of low IgA gammopathy, pyoderma gangrenosum developed on both knees, following vesiculobullous lesions. Similarly, Choulot and Saint Martin [22] described pyoderma gangrenosum in a patient with low IgA syndrome.

In a bit older references, Sánchez Yus et al. [23] showed a case of pyoderma gangrenosum with myeloma IgA lambda and deficiency of cellular immunity and Barrière et al. [24] presented a child with congenital hypogammaglobulinemia and pyoderma gangrenosum.

We have presented a case of co-existence of primary variable immunodeficiency and pyoderma gangrenosum, which despite typical history, was not recognized for more than a year. In our patient, despite difficulties with therapy, we reached improvement of skin changes and probably it would be completely cured.

\section{References}

1. Braun-Falco O, Plewig G, Wolff HH, et al. Pyoderma gangrenosum. In: Dermatology. Braun-Falco O, Plewig G, Wolff $\mathrm{HH}$, et al. (eds.) Czelej, Lublin 2002; 858-61.

2. Duke G, Al Samaraee A, Husain A, et al. Pyoderma gangrenosum: a rare cause of breast ulceration. Ochsner I 2012; 12: $155-8$

3. Kosari P, Feldman SR. Treatment-resistant pyoderma gangrenosum. Dermatol Online J 2012; 18: 8.

4. Schadt CR, Callen JP. Management of neutrophilic dermatoses. Dermatol Ther 2012; 25: 158-72.

5. Dua J, Elliot E, Bright P, et al. Pyoderma gangrenosum-like ulcer caused by Helicobacter cinaedi in a patient with $x$ linked a gammaglobulinaemia. Clin Exp Dermatol 2012; 37: 642-5.

6. Omiya W, Ujiie H, Akiyama M, et al. Coexistence of pustular and vegetative pyoderma gangrenosum in a patient with myelodysplastic syndrome. Eur J Dermatol 2012; 8: 146-9.

7. Neiderer K, Martin B, Hoffman S. A mechanically powered negative pressure device used in conjunction with a bioengineered cell-based product for the treatment of pyoderma gangrenosum: a case report. Ostomy Wound Manage 2012; 58: 44-8

8. Fraccalvieri M, Fierro MT, Salomone M, et al. Gauze-based negative pressure wound therapy: a valid method to manage pyoderma gangrenosum? Int Wound I 2012; 14: 127-34.

9. Andrade P, Brites MM, Figueiredo A. Synchronous pyoderma gangrenosum and inflammatory bowel disease, healing after total proctocolectomy. An Bras Dermatol 2012; 87: 637-9.

10. Andrisani G, Guidi L, Papa A. A case of pyoderma gangrenosum with ulcerative colitis treated with combined approach: infliximab and surgery. J Crohns Colitis 2012; 10: 467-72.

11. Mooij JE, van Rappard DC, Mekkes JR. Six patients with pyoderma gangrenosum successfully treated with infliximab. Int J Dermatol 2012; 18: 234-9.

12. Kim FS, Pandya AG. The use of etanercept in the treatment of peristomal pyoderma gangrenosum. Clin Exp Dermatol 2012; 37: 442-3. 
13. Wolfe CM, Green WH, Cognetta AB. Atypical pyoderma gangrenosum of the dorsal hand mimicking squamous cell carcinoma. J Hand Surg Am 2012; 37: 1835-8.

14. Maksimovic L, Duriez P, Lascaux-Cametz AS, et al. Erythema elevatum diutinum associated with pyoderma gangrenosum in an HIV-positive patient. Ann Dermatol Venereol 2010; 137: 386-90

15. Kreuter A, Gambichler T, Hoffmann K, et al. Association of HIV infection, pyoderma gangrenosum and psoriasis. Acta Derm Venereol 2002; 82: 150-2.

16. Nord KM, Pappert AS, Grossman ME. Pyoderma gangrenosum-like lesions in leukocyte adhesion deficiency I treated with intravenous immunoglobulin. Pediatr Dermatol 2011; 28: 156-61.

17. Hinze CH, Lucky AW, Bove KE, et al. Leukocyte adhesion deficiency type 1 presenting with recurrent pyoderma gangrenosum and flaccid scarring. Pediatr Dermatol 2010; 27: 500-3.

18. Elenberg Y, Shani-Adir A, Hecht Y, et al. Pyoderma gangrenosum after bone marrow transplantation for leukocyte adhesion deficiency type 1. Isr Med Assoc J 2010; 12: 119-20.

19. Bedlow AJ, Davies EG, Moss AL, et al. Pyoderma gangrenosum in a child with congenital partial deficiency of leucocyte adherence glycoproteins. Br J Dermatol 1998; 139: 1064-7.

20. Paller AS, Sahn EE, Garen PD, et al. Pyoderma gangrenosum in pediatric acquired immunodeficiency syndrome. J Pediatr 1990; 117: 63-6.

21. Carsuzaa F, Pierre C, Dubegny M. Pyoderma gangrenosum and IgA gammopathy. Association with atrophic gastritis. Ann Dermatol Venereol 1989; 116: 707-13.

22. Choulot JJ, Saint Martin J. Pyoderma gangrenosum associated with IgA deficiency. Ann Pediatr (Paris) 1987; 34: 252.

23. Sánchez Yus E, Cabré J. Pyoderma gangrenosum (PG) with myeloma IgA lambda and deficiency of cellular immunity. Actas Dermosifiliogr 1980; 71: 35-40.

24. Barrière H, Litoux P, Stalder JF, et al. Pyoderma gangrenosum associated with congenital hypogammaglobulinemia. Ann Dermatol Venereol 1979; 106: 695-6. 